\title{
Intracellular activation of complement C3 in Paneth cells improves repair of intestinal epithelia during acute injury
}

\author{
Jian Zhang ${ }^{\ddagger}$, , Jinning Ye ${ }^{\ddagger 1}$, Yufeng Ren ${ }^{\ddagger}{ }^{2}$, Jidong Zuo ${ }^{1}$, Weigang Dai ${ }^{1}$, Yulong He ${ }^{1,3}$, Min \\ $\operatorname{Tan}^{1}$, Wu Song ${ }^{1,3}$ \& Yujie Yuan*,1,3 \\ ${ }^{1}$ Center of Gastrointestinal Surgery, The First Affiliated Hospital, Sun Yat-sen University, Guangzhou, 510080, PR China \\ ${ }^{2}$ Department of Radiation Oncology, The First Affiliated Hospital, Sun Yat-sen University, Guangzhou 510080, PR China \\ ${ }^{3}$ Center of Gastric Cancer, Sun Yat-sen University, Guangzhou 510080, PR China \\ *Author for correspondence: Tel.: +86 208775576 68718; Fax: +86 208733 1059; condor.yyj@gmail.com \\ ${ }^{\ddagger}$ Authors contributed equally
}

\begin{abstract}
Aim: To explore whether Paneth cells (PCs) and complement system collaborate in the repair of enteric epithelia during acute gastrointestinal injury (AGI). Methods: Wild-type C57BL/6 mice were employed to induce AGI by performing colon ascendens stent surgery, with sham-operated as control. Exogenous $\mathrm{C} 3$ treatment was applied at 6-h postsurgery. After $48 \mathrm{~h}$, overall survival, intestinal damage severity, and C3 intracellular activation were assessed in both epithelial cells and PCs. Results: AGI caused a high mortality, while C3 therapy significantly attenuated epithelial damages and improved survival. Besides, exogenous C3 in vitro enhanced the proliferation and activity of PCs. Importantly, intracellular C3 activation was observed inside of PCs under C3 co-stimulation in vitro. Conclusion: C3 immunotherapy might play a valuable role in turnover of gut epithelia through intracellular activation in PCs.
\end{abstract}

First draft submitted: 4 September 2018; Accepted for publication: 1 October 2018; Published online: 23 November 2018

Keywords: abdominal infection • complement C $\bullet$ gut epithelia $\bullet$ immunotherapy $\bullet$ intestinal injury $\bullet$ intracellular activation • lysozyme $\bullet$ Paneth cells $\bullet$ repair and regeneration $\bullet$ survival

The intestinal mucosa provides both protective and nutritional effects for life events. As a first line of defense, numerous factors, such as pathogenic invasion and allergic reaction, could eventually lead to intestinal mucosa damage and dysfunction, which is called acute gastrointestinal injury (AGI) [1]. Intra-abdominal infection (IAI), which is a common pathological problem and can be induced experimentally by a cecal puncture or colon ascendens stent model, has been confirmed to be strongly correlated with severe peritonitis and rapid intestinal injury [2,3]. Acute injury to local intestines and remote organs often contributes to a high mortality of 60-90\% [4].

The complement system is an indispensable mediator of acute injury in various organs, such as the gut, heart, kidney, liver and lung [5]. It could initiate cascade reactions to pathogenic invasion or internal tissue damage via at least four pathways [6]. Complement C3 is a crucial molecule in such complement cascades [7]. In our preliminary study, extensive complement activation followed by circulating C3 depletion has been observed in a mouse model of severe IAI. In addition, administration of exogenous C3 at an early stage of infection could postpone C3 depletion and enhance immunity responses to bacterial infection [8].

Paneth cells (PCs), first described by J Paneth and G Schwalbe, are located mainly in the bottoms of crypts of Lieberkuhn, beneath intestinal villi [9]. The role of PCs in innate intestinal immunity is universally recognized due to their characteristic granules, such as $\alpha$-defensins and lysosomes [10,11]. Emerging evidence indicates that PCs could actively sense enteric bacteria and play a vital role in maintaining host-microbial homeostasis of the intestinal epithelia [11]. Intestinal PCs are the earliest responders to bacterial infection and enhance innate immune protection from enteric pathogens [12]. Dysfunction of PCs biology contributes to various severities of gut inflammation. However, it is still unknown whether PCs and the complement system collaborate in intestinal epithelial function, especially during acute intestinal injury. 
Recently, several immune cells were found to produce $\mathrm{C} 3$ and self activate intracellularly by an internal protease, cathepsin L [13-15]. Accordingly, we propose a hypothesis that intracellular C3 activation in PCs would upregulate activities of PCs and reduce enteric epithelia damage during an AGI. This study is designed to unravel the relationship between the complement system and PCs and assist in finding a possible mechanism of gut epithelia repair during an acute injury.

\section{Materials \& methods}

\section{Experimental animals \& models}

Wild-type 8 to 10-week-old female C57BL/6 mice (weight 20-25 g) were purchased from the Laboratory Animal Center (Jiangxi Province, PR China). All mice were maintained in standard wired cages under a constant 12-h light and dark cycle and a specific-pathogen-free environment at $20-23^{\circ} \mathrm{C}$. The mice were allowed ad libitum access to water and foods, with a 1 -week rest required before the start of experiments. Colon ascendens stent peritonitis procedures were performed under general anesthesia $(0.5 \%$ pentobarbital sodium, $20 \mu \mathrm{l} / \mathrm{g}$, ip. $)$ to induce AGI as previously described [2]. Briefly, a prepared stent $\left(5 \mathrm{~mm} \times 2.0 \mathrm{~mm}^{2}\right.$, Venflon; BOC, Ohmeda AB, Helsingborg, Sweden) was stitched through the antimesenteric wall into the proximal lumen of the ascending colon and fixed at about $1 \mathrm{~cm}$ distant to the ileocecal valve. Afterward, stool was milked from the cecum into the stent until a drop of stool appeared. For a control, a sham operation with a stent fixed outside the ascending colon was applied. After those operations, mice were brought back to the cages, with free access to water for the next $48 \mathrm{~h}$.

\section{Exogenous C3 administration \& study design}

The mice were randomly divided into four groups as follows: AGI, C3+ group ( $\mathrm{n}=15)$; AGI, C3- group $(\mathrm{n}=15)$; Sham, C3+ group ( $\mathrm{n}=15)$; Sham, C3- group $(\mathrm{n}=15)$. After $6 \mathrm{~h}$ of surgery, a single dose of $1 \mathrm{mg}$ exogenous complement C3 protein (human source, MybioSource, CA, USA), diluted in $200 \mu$ phosphate-buffered saline (PBS) plus $0.1 \%$ bovine serum albumin under sterile conditions, was intraperitoneally injected in the two C3+ groups, whereas only $200 \mu \mathrm{l}$ PBS plus $0.1 \%$ bovine serum was injected at the same time-point in both C3-groups. Of note, additional experiment for the observation of 48-h survival following intestinal injury were performed among all groups ( $\mathrm{n}=15$ /group) before the following experiments.

\section{Evaluation of intestinal injury \& C3 intracellular activation}

After $24 \mathrm{~h}$ of surgery, five alive mice in each group were sacrificed by neck dislocation, and the ileum nearest the stented colon was harvested and cut into $1 \times 1 \times 2 \mathrm{~mm}^{3}$ segments and immediately prefixed with 3\% glutaraldehyde- $4 \%$ paraformaldehyde- $0.1 \mathrm{~mol} / \mathrm{l}$ phosphate buffer $(\mathrm{pH} 7.2)$ at $4{ }^{\circ} \mathrm{C}$ for $2 \mathrm{~h}$. Then, the samples were washed and postfixed with $1 \%$ osmium tetroxide at $4^{\circ} \mathrm{C}$ for $1.5 \mathrm{~h}$. After gradient dehydration with alcohol and acetone, the samples were embedded in epoxy resin 618 and then cut into $60-\mathrm{nm}$ slides. The slides were stained with uranyl acetate and lead citrate and photographed under a transmission electron microscope (JEOL-100 CX) at $80 \mathrm{kV}$. For routine histopathological analysis, the harvested samples were sectioned and stained with hematoxylin and eosin once tissues were fixed with paraformaldehyde and embedded in paraffin.

The intestinal integrity after acute injury was evaluated blindly with the following scoring system: score 0 , no damage; score 1, subepithelial space at villous tip; score 2, loss of mucosal lining of the villous tip; score 3, loss of less than half of villous structure; score 4 , loss of more than half of villus structure and score 5 , transmural necrosis $[3,16]$.

Immunofluorescent confocal microscopy was utilized to detect C3 deposition in intestinal epithelia. A FITCconjugated goat anti-C3 Ab (dilution 1:600, Abcam, ab200999, Cambridge, MA, USA) was used to detect C3, as well as $\mathrm{C} 3 \mathrm{a}, \mathrm{C} 3 \mathrm{~b}$ and $\mathrm{C} 3 \mathrm{c}$ fragments. On the following day, frozen sections from the described above-harvested intestine, were incubated with the $\mathrm{Ab}$ for $1 \mathrm{~h}$ at room temperature, washed three times with PBS for $10 \mathrm{~min}$ each, coated with antifade mounting medium (Molecular Probes), covered and analyzed with a Zeiss confocal microscope (Laser Scanning Microscope 710, Carl Zeiss, Göttingen, Germany) as previously described by using the same parameters for each tissue examined [17]. Of note, optical sectioning was applied to remove background fluorescence and to localize intracellular staining. Controls with streptavidin-conjugated FITC without C3 antibody stained were processed above to determine nonspecific binding (Supplementary Figure 1). 


\section{PCs harvest \& identification}

After $6 \mathrm{~h}$ of recovery from surger, alive mice were sacrificed via cervical dislocation, and PCs were harvested from crypts of the ileum, as previously described [18]. In brief, crypts from the ileum were mainly collected from mixed intestinal fragments through a $70-\mu \mathrm{m}$ mesh filter. Next, the crypt suspensions were marked with an antibody cocktail consisting of CD45-PE, EPCAM-APC (eBioscience, CA, USA), CD31-PE, Ter119-PE, CD31-PE, Ter119-PE and CD24-Pacific Blue (BioLegend, CA, USA), and resuspended with SEME/7-aminoactinomycin D (7-AAD) solution (1:500 dilution), followed by flow cytometry selection and analysis using a forward and side scatter gating strategy (BD Biosciences, San Jose, CA, USA). PCs were isolated as CD24 $4^{\text {hi }}$ Epcam ${ }^{+}$CD $31^{-}$Ter $119^{-} \mathrm{CD}^{-} 5^{-}$7-AAD ${ }^{-}$ cells, with the percentage in the crypt suspensions recorded. Dead cells were abandoned for subsequent studies, using the viability dye 7-AAD.

\section{Culture of isolated PCs with exogenous $\mathrm{C3}$}

After $48 \mathrm{~h}$ of in vitro culture at 30-50 $\mathrm{l}$ drops of matrigel (BD Bioscience, San Jose, CA, USA), the activity of isolated PCs was evaluated through immunofluorescence and Western blot methods, respectively [19]. Briefly, mouse anti-PAP III antibody (1:1000) was used to mark PCs, nuclei were visualized with 4'6-diamidino-2-phenylindole (DAPI) staining for fluorescence. For Western blot analysis, antimouse RegIII- $\gamma$ was used to anchor PCs and quantify their expression. Lysozyme, a secretory marker of PCs, was measured to detect the activity of PCs as previously reported [20-22]. Moreover, intracellular activation of complement components, such as C3, C3a and cathepsin L proteins, were detected by Western blot method as previously performed [23].

\section{Statistical analysis}

Measurement data were reported as the mean \pm SEM (standard error of the mean), unless stated otherwise. Variables were compared by student's $t$-test when groups were distributed normally. Otherwise, Mann-Whitney $\mathrm{U}$ test was performed for group comparison. Specifically, comparison between C3+ group and C3-group was completed with an unpaired test. A log-rank (Mantel Cox) test (minimum $\mathrm{n}=10)$ was used to compare survival curves among the various C3 treatment groups. Statistical analysis was performed using Prism Software (version 7.0 for windows, GraphPad Software, Inc., CA, USA). A p-value with a two-tail test of $<0.05$ was considered as statistically significant.

\section{Results}

\section{Exogenous C3 supplementation improves survival following AGI}

After $24 \mathrm{~h}$ of surgery, all mice developed severe IAI and AGI, with the exception of the sham-operated mice. Within $48 \mathrm{~h}$ of the postoperative observation, the overall survival rate in the AGI-induced mice was significantly poorer compared with the Sham-operated mice $(24.6$ vs $87.5 \%$; $\mathrm{p}<0.001)$. To be specific for AGI-induced mice, the overall survival in mice with exogenous $\mathrm{C} 3$ supplementation (AGI, C3+ group) was markedly improved compared with mice without C3 treatment (AGI, C3- group; $\mathrm{p}<0.01$; Figure 1).

\section{Exogenous $\mathrm{C} 3$ administration attenuates intestinal barrier damage from AGI}

As mentioned above, the terminal ileum nearest the stent site was harvested to evaluate the integrity of the intestinal barrier (Figure 2). The induced AGI led to massive intestinal barrier damage, such as mucosal collapse, loss of villi or epithelial cells and massive infiltration of neutrophils (Figure 2B). Administration of exogenous C3 at 6-h postoperation significantly attenuated such lesions from AGI (Figure 2C). The average histopathology score in the Sham, C3- group was similar to the Sham, C3+ group (mean, 0.3 vs $0.3 ; \mathrm{p}=0.976$ ). In contrast, the average score in the AGI, C3- group was much greater than that in the AGI, C3+ group (mean, 2.5 vs 1.2; $\mathrm{p}<0.001$; Figure 2D). Additionally, the tight junction between intestinal epithelial cells was markedly improved with the treatment of exogenous C3 administration (Figure 3).

\section{C3 deposition in intestinal epithelial cells contributes to intestinal injury}

To explore the role of complement activation in the initiation of acute intestinal injury, the intracellular deposition of C3 and its activators in intestinal epithelia were evaluated 24-h after surgery. Villus damage combined with extensive C3 deposition in intestinal epithelial cells was observed in the AGI, C3- group. However, exogenous C3 treatment 6-h postoperation did not cause more intracellular deposition of C3 or other active effectors but was associated with a remarkable decrease in intestinal villus damage when compared with non-C3 treatment 


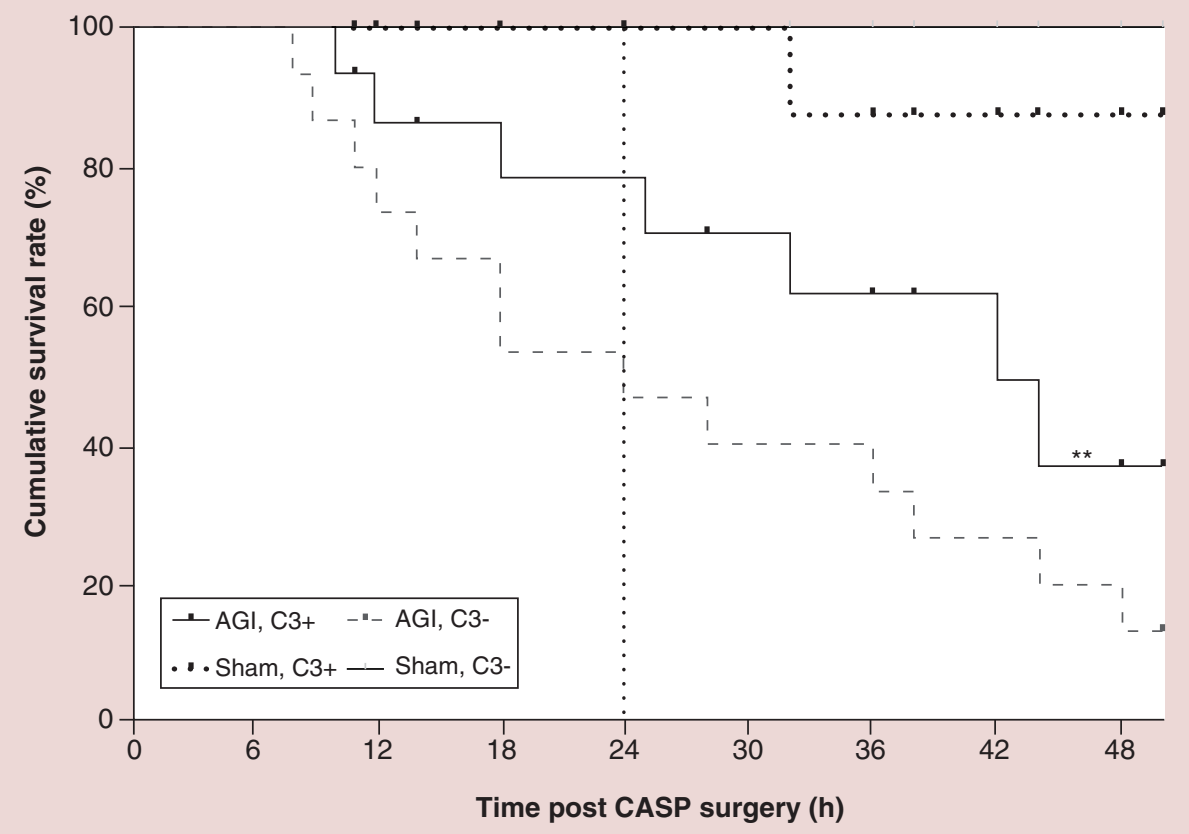

Figure 1. Survival curve in the C57BL/6 mice model of acute gastrointestinal injury. Groups are depicted as indicated ( $n=15$ /group). The Kaplan-Meier method was employed for the 48-h survival calculation. The overall survival rates were 100 and $87.5 \%$ in the Sham, C3- and Sham, C3+ groups, respectively. Whereas survival rate in the AGI, C3- group was $46.7 \%$ at $24 \mathrm{~h}$, but decreased to $13.3 \%$ at 48 -h postoperation. Exogenous administration of C3 protein at 6-h postoperation improved survival significantly, with $78.3 \%$ at $24 \mathrm{~h}$ and $37.0 \%$ at $48 \mathrm{~h}$. $* * p<0.01$ versus AGI, C3- group.

AGI: Acute gastrointestinal injury; CASP: Colon ascendens stent peritonitis.

(Figure 4). Taken together, these data indicate that intracellular C3 deposition in intestinal epithelia contributes to the development of tissue damage, which could be resolved with exogenous C3 treatment.

\section{C3 enhances the function and proliferation of Paneth cells during intestinal injury}

The function of PCs during an acute gut injury was evaluated at 0,12 and $48 \mathrm{~h}$ after surgery, respectively. Without the treatment of exogenous $\mathrm{C} 3$, the production of lysozymes was gradually increased, but declined quickly $12 \mathrm{~h}$ after the surgery. To address whether sufficient circulating C3 is responsible for the function of PCs during intestinal injury, exogenous C3 was supplied to mice at 6-h postoperation. As expected, the production of lysozymes in C3-treated mice was also increased initially after surgery, and sustained significantly high levels around bottoms crypts of the ileum 48-h postoperation, compared with saline controls (Figure 5). On the other hand, the amount of isolated PCs was also remarkably increased in C3-treated mice at $24 \mathrm{~h}(7.8$ vs $5.7 \% ; \mathrm{p}=0.032)$ a;nd $48 \mathrm{~h}$ ( 12.6 vs $7.6 \% ; p=0.003$ ) postoperation, respectively, compared with saline-treated mice (Figure 6). These findings demonstrate that exogenous $\mathrm{C} 3$ could enhance the function and proliferation of PCs during experimental acute intestinal injury.

\section{Intracellular activation of C3 is responsible for activation of Paneth cells}

To further explore the relationship between PCs and C3 activation, isolated PCs from Sham-operated mice were cultured in vitro with or with purified C3 protein. After $48 \mathrm{~h}$ of culturing, intracellular deposition of C3 and its active components was significantly enhanced in C3-stimulated PCs, compared with normal cultured PCs (Figure 7A). Moreover, the production of PAP III protein from isolated PCs was markedly enriched under C3 stimulation, compared with normal culture medium (Figure 7B). Moreover, the release of RegIII- $\gamma$ from cultured PCs was significantly increased with exogenous $\mathrm{C} 3$ stimulation, compared with normal culture medium (Figure 7C). 

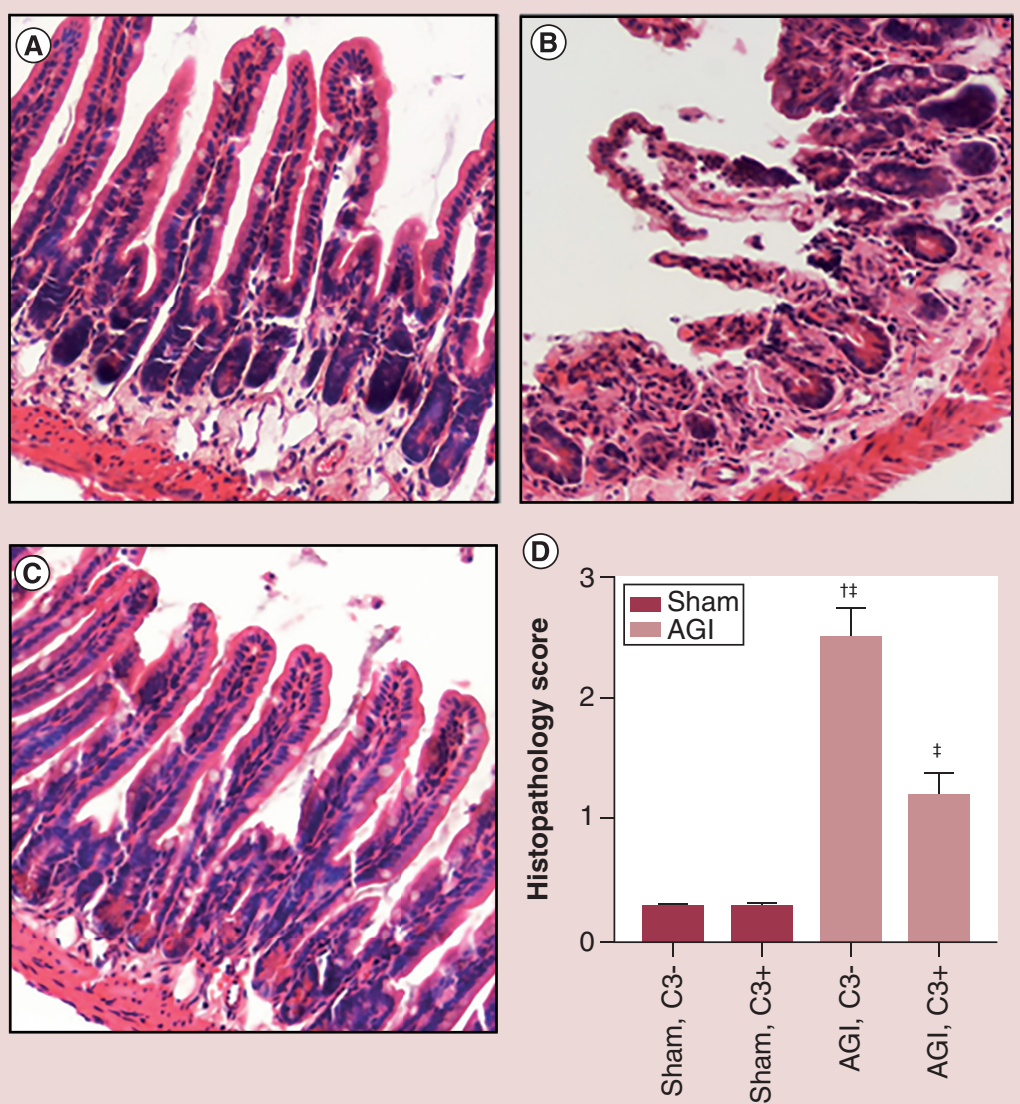

Figure 2. Photomicrograph indicating morphological changings of intestinal integrity during acute intestinal injury. (A) A typical hematoxylin and eosin staining image of a section of terminal ileum from a Sham-operated mouse at $24 \mathrm{~h}$ postsurgery. (B) Photomicrograph of a section of ileum from an AGl-induced mouse without C3 injection $24 \mathrm{~h}$ after surgery; (C) a typical image from an injured mouse, but treated with exogenous C3 injection $24 \mathrm{~h}$ after surgery. Original magnification $100 \times$. The histopathological score of harvested ileum at 24-h postsurgery in each group (D) was expressed as the mean \pm SEM $(n=5)$ and compared by one-way analysis of variance (ANOVA). ${ }^{\dagger} p<0.001$ versus $A G I, C 3+$ group; ${ }^{\ddagger} p<0.001$ versus two sham-operated groups.

AGI: Acute gastrointestinal injury; SEM: Standard error of the mean.

Collectively, this data support that exogenous C3 could directly improve intracellular activation of C3 in PCs and enhance its activities.

\section{Discussion}

In the current study, the role of exogenous $\mathrm{C} 3$ supplementation in reducing intestinal damage from acute injury has been explored, followed by a possible investigation of the mechanism. The findings suggest that massive C3 deposition in intestinal epithelium was associated with tissue damage during an acute injury but could be inhibited by exogenous C3 administration through intracellular activation of C3 in PCs at the bottom crypts of the ileum. The exogenous C3 supplementation is essential for enhancing the function and proliferation of PCs during AGI. To the best of our knowledge, this study is the first investigation using animal models to correlate intracellular C3 activation with PCs activity during acute gut injury.

It is well known that the main effect of the complement system is the induction of pathogen-related and -modulated cascade reactions, which are responsible for the lysis of targeted cells and the protection of the host from infection [24]. Such protective effects are realized mainly through three recognized pathways: the classical, alternative and lectin pathways [25]. Of note, those pathways for activation also play role in killing other cells, such as a liver-derived serum effector cascade. However, the newly discovered intracellular activation represents a 

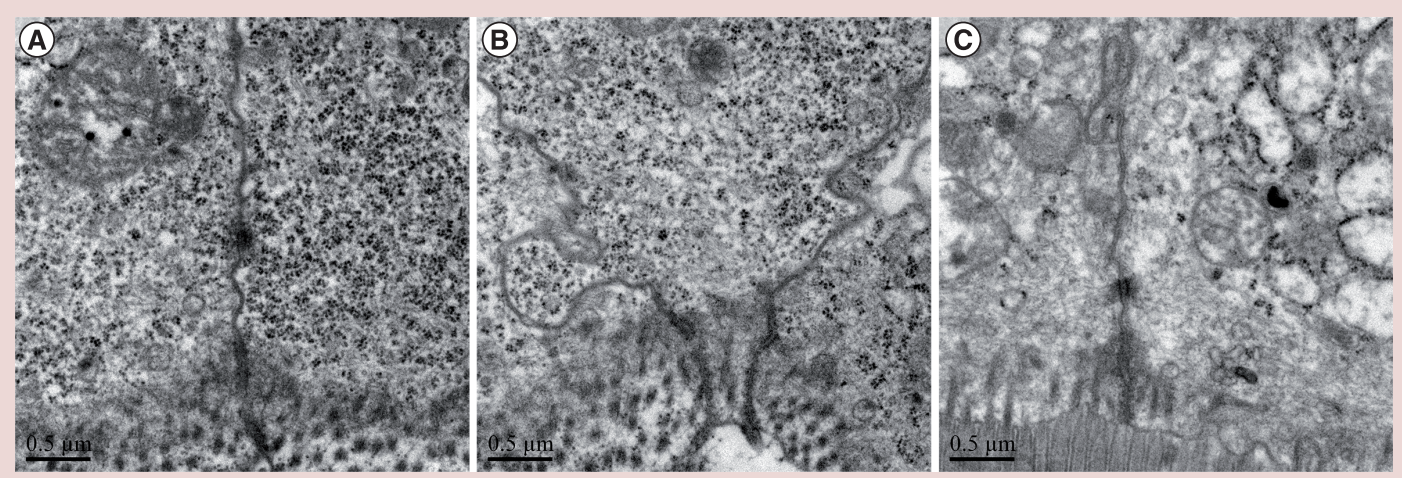

Figure 3. The intracellular tight junction between intestinal epithelial cells during acute intestinal injury. Transmission electron microscopy was employed to measure the gap of the injured tight junction between mucosal cells. (A) The TJ in a Sham-operated mouse $24 \mathrm{~h}$ after surgery. TJ gap $=249.3 \mu \mathrm{m}$; (B) The TJ in an AGI-induced mouse without exogenous C3 treatment $24 \mathrm{~h}$ after surgery. TJ gap $=448.5 \mu \mathrm{m}$; (C) The TJ in an AGI-injured mouse treated with C3 injection 6-h postoperation, was improved compared with the mouse without C3 treatment. TJ gap $=257.2 \mu \mathrm{m}$. White arrows stand for TJ gap; Scale bars, $2 \mu \mathrm{m}$. Representative images of $\mathrm{n}=5$ independent experiments. AGI: Acute gastrointestinal injury; TJ: Tight junction.
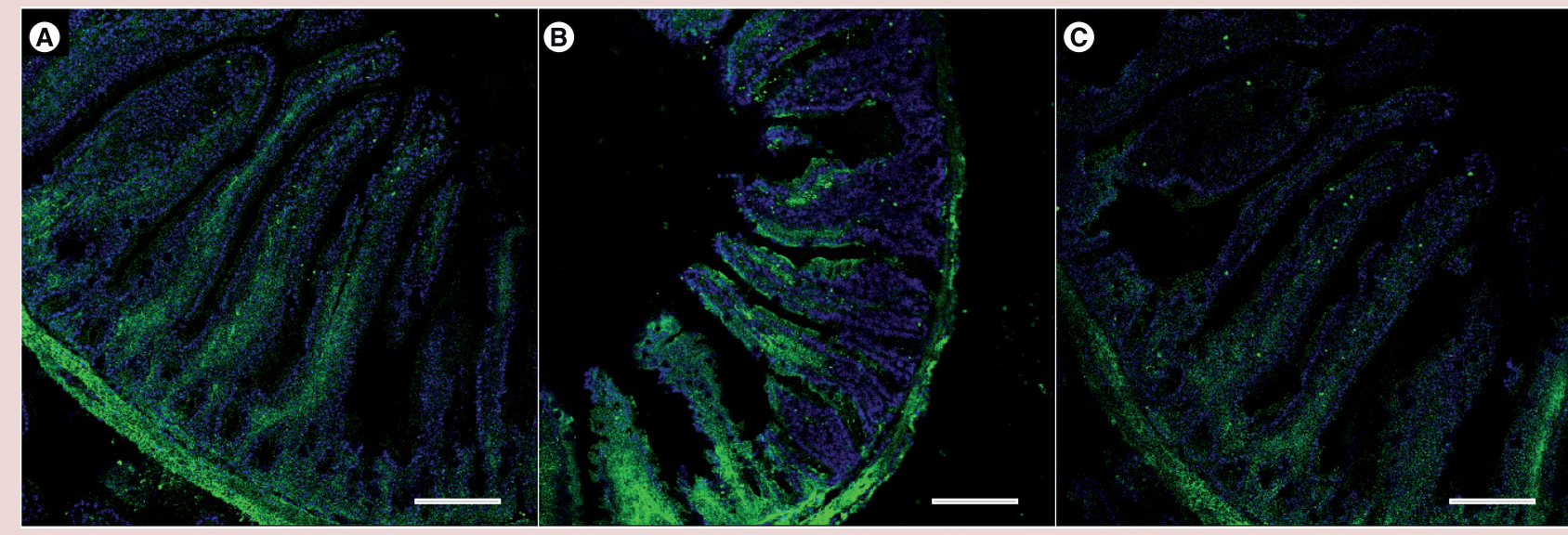

Figure 4. Intracellular deposition of C3 in intestinal epithelial cells during acute intestinal injury. Immunofluorescence confocal micrographs were employed to explore intracellular deposition of C3 in terminal ileum. Samples were harvested from Sham-operated mice (A) or AGI-induced mice (B \& C) at 24-h postoperation. Exogenous C3 treatment 6-h postoperation reduced the intestinal injury and promoted the regeneration of intestinal epithelium (C), compared with non-C3 treatment (B). The green fluorescent dots indicate intracellular C3 deposition in the epithelia, with blue staining indicating the nucleus by DAPI method. Original magnification $200 \times$ AGI: Acute gastrointestinal injury; DAPI: 4'6-diamidino-2-phenylindole.

paradigm shift in the conventional viewpoint to complement biology, which plays an essential role in the regulation of cellular homeostasis and own survival [26-28]. More importantly, such activation is not restricted to immune cells only but is also likely to have broad physiological significance $[28,29]$.

In our study, C3 deposition in PCs is noticeably observed at the base of crypts during AGI, and for the first time, the enhanced proliferation of PCs has been shown by exogenous C3 stimulation. Meanwhile, intestinal epithelial tight junction permeability seems to strengthen accordingly through $\mathrm{C} 3$ treatment. It might be understandable, since PCs are required for limiting penetration of the gut barrier by both commensal and pathogenic bacteria [11]. In addition, PCs are also indispensable in providing essential niche signals to enteric stem cells [30,31], which would indirectly influence the growth of intestinal epithelial cells [19,32]. 


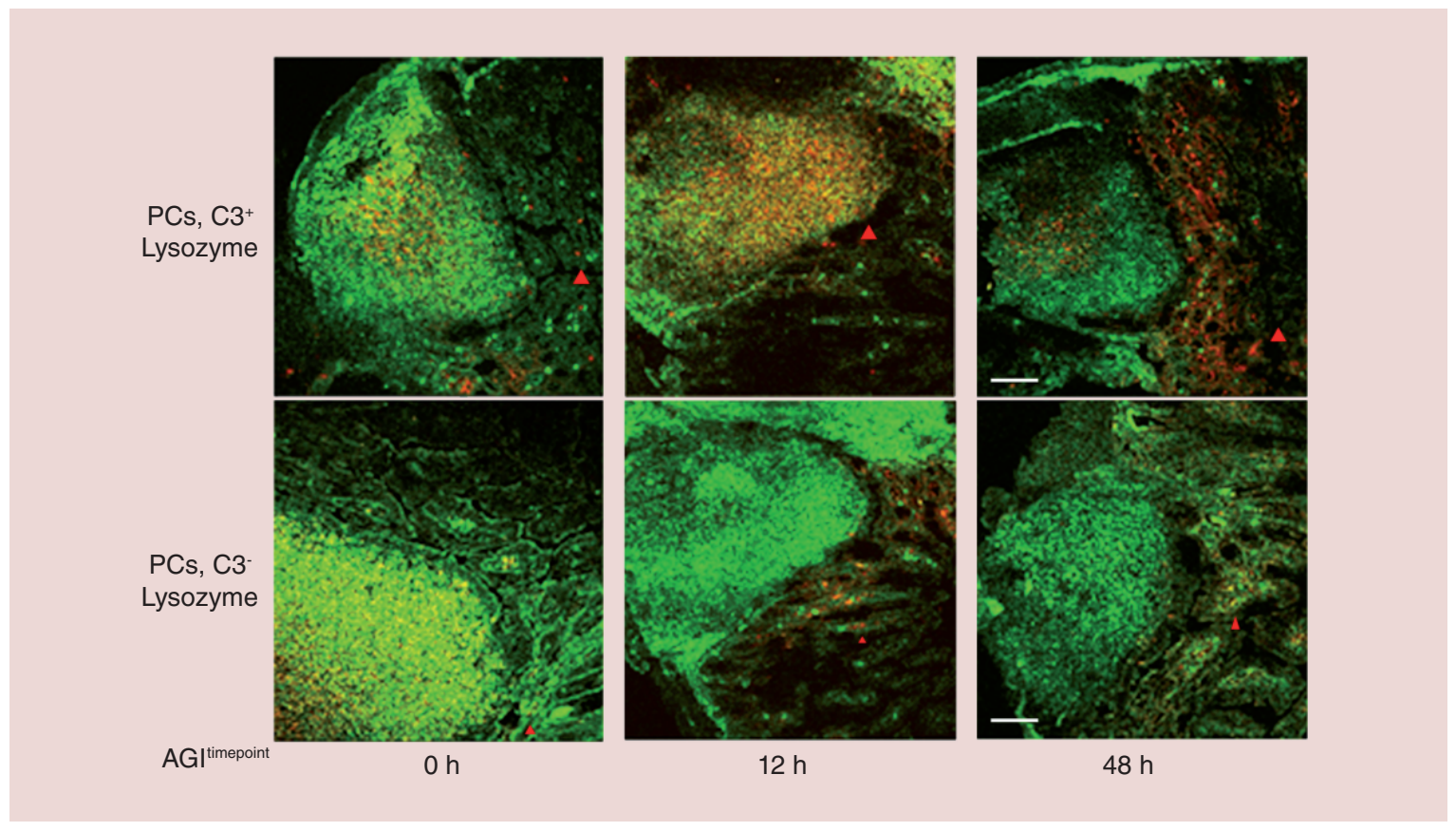

Figure 5. The productive activity of Paneth cells during acute intestinal injury. Immunofluorescence confocal micrographs were used to investigate the relationship between PCs productive function and C3 deposition. The involved ileum segments were harvested from AGI-induced mice at 0-, 12- and 48-h postsurgery, respectively. The red-stained lysozymes, which are produced by PCs, indicate its productive activity, whereas green fluorescent dots stand for C3 deposition. Red triangles indicate the bottoms of crypts in the intestinal epithelium. Exogenous C3 treatment enhances the activities of PCs, especially at 48-h postoperation, during an acute injury. Scale bars, $200 \mu \mathrm{m}$. Representative images of $n=5$ independent experiments.

AGI: Acute gastrointestinal injury; PC: Paneth cell.

Apart from C3 activation in PCs, massive C3 deposition in intestinal epithelia is also detected at the early stage of AGI, followed by extensive epithelial loss. Such uncontrolled C3 intracellular activation is believed to contribute to subsequent tissue damage [33]. The possible mechanism of enteric epithelia injury might be aroused from AGI-induced mesenteric ischemia since the villus epithelium is quite vulnerable for ischemia/reperfusion injury [1,34]. There is plenty of evidence that numerous complement components, such as C3, C5, C3a, C5a, C3aR and CR2, are involved in the various severities of the mesenteric ischemia model and lead to local intestinal and remote organ injury [35-37]. In our study, the regional deposition of C3 and its effectors is markedly reduced once exogenous C3 treatment started at the early stage of AGI. It might be related to the enhanced function of PCs, which is responsible for improved microbiota in intestine [38,39].

Several shortcomings of this study should be addressed here. First, we failed to explore the possible pathway of intracellular $\mathrm{C} 3$ activation in the regulation of PCs and their active effectors during AGI. Further studies designed to find a signaling pathway to link them together are still underway. Second, another control group employing C3-knockout mice should be required to further confirm the role of intracellular C3 activation in PCs but is unavailable in our study due to limited animal resources. Alternative groups with circulating C3 depletion by cobra venom factor may be possibly realized in future works. Moreover, the evaluation on histological changes appears subjective, and the intestinal epithelial changes should be evaluated with a more objective manner. Third, the relationship between C3 deposition in intestinal epithelia and PCs is still unknown based on the current study. It seemed that activated PCs induced by C3 intracellular activation could inhibit the deposition of C3 in epithelial cells during acute injury. Another more in-depth study exploring their internal connection is indispensable to reveal the repair mechanism of gut epithelia lesions. Last, our findings originated from a specific infection model, which produced a secondary intestinal injury after severe infection. Therefore, it would be insufficient to suggest such findings to intestinal injury in general, and a more accurate model of AGI should be required for validation.

In this study, the anti-C3 antibodies used for examining PCs react to both human and mouse $\mathrm{C} 3$. This complicates the interpretation of some results as the intracellular C3 may be either due to production of internal C3 by the cells 
(A)

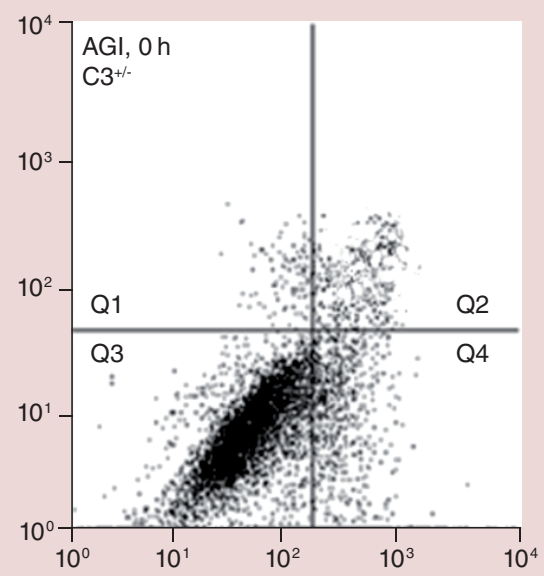

(B)

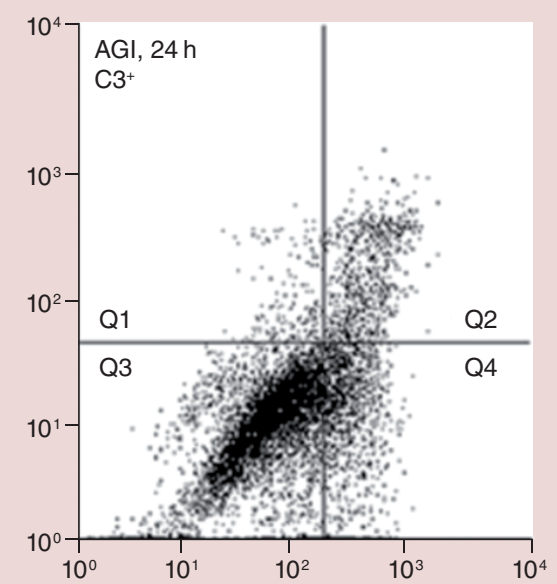

(D)

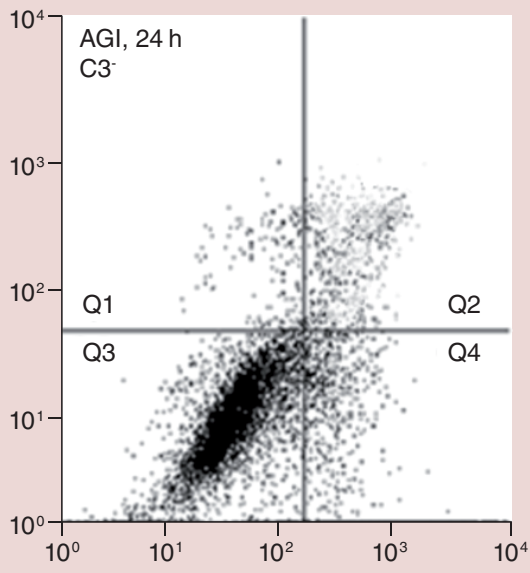

(F)

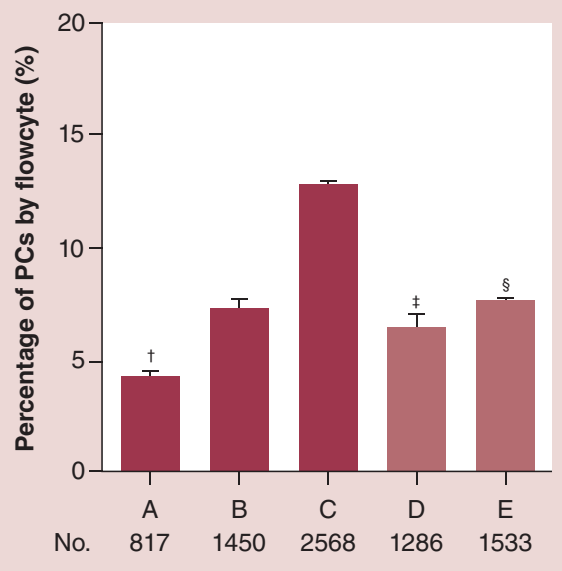

(C)

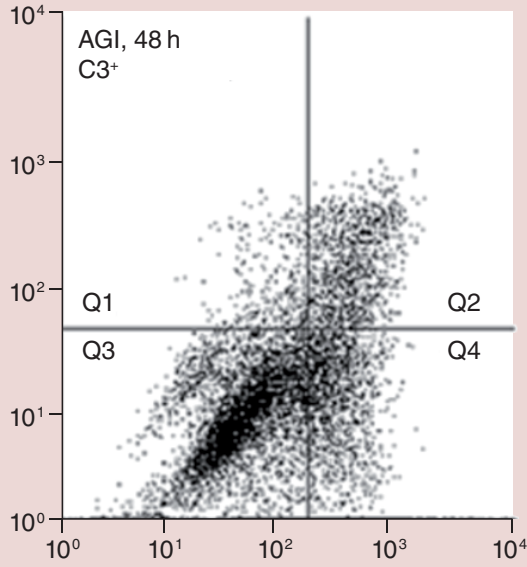

(E)

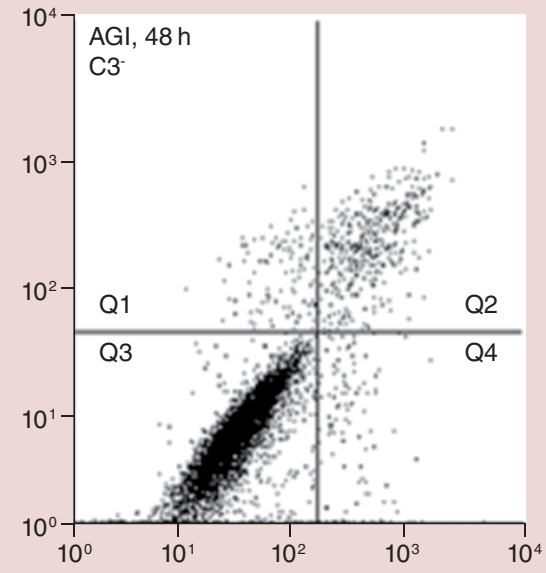

Figure 6. The proliferation of Paneth cells during acute intestinal injury. Flow cytometry analysis was employed to explore the percentage of PCs in separated crypts suspension. Samples were harvested from AGI-induced mice at $0 \mathrm{~h}$ (A), $24 \mathrm{~h}$ (B \& D) and $48 \mathrm{~h}$ (C \& E) postoperation, respectively. The $\mathrm{x}$ - and y-axis labels (A-E) were CD24-FITC-H and EpCAM-APC-H, respectively. Relative percentages of PCs in Q2 of each flow dot plot (20,000 cells per sample) were compared via histograms $(F)$, with error bars standing for the mean \pm SEM $(n=5)$. AGI promotes the proliferation of PCs at an early stage of injury, which is further enhanced by exogenous $\mathrm{C} 3$ administration at 6-h postoperation. ${ }^{\dagger} \mathrm{p}$ $<0.05$ versus group B-D; ${ }^{\ddagger} p<0.05$ versus group $B ;{ }^{\S} p<0.01$ versus group $C$. AGI: Acute gastrointestinal injury; PC: Paneth cell; SEM: Standard error of the mean. 
(A)

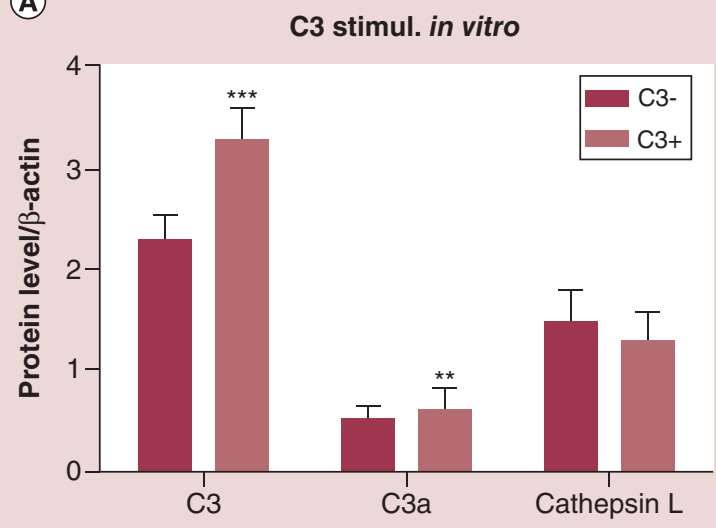

(B) PAP III/DAPI

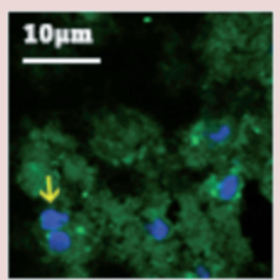

C3-

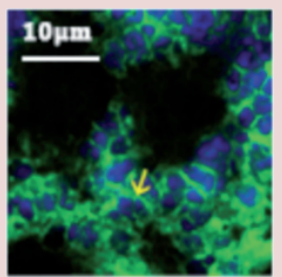

$\mathrm{C} 3+$
(C)
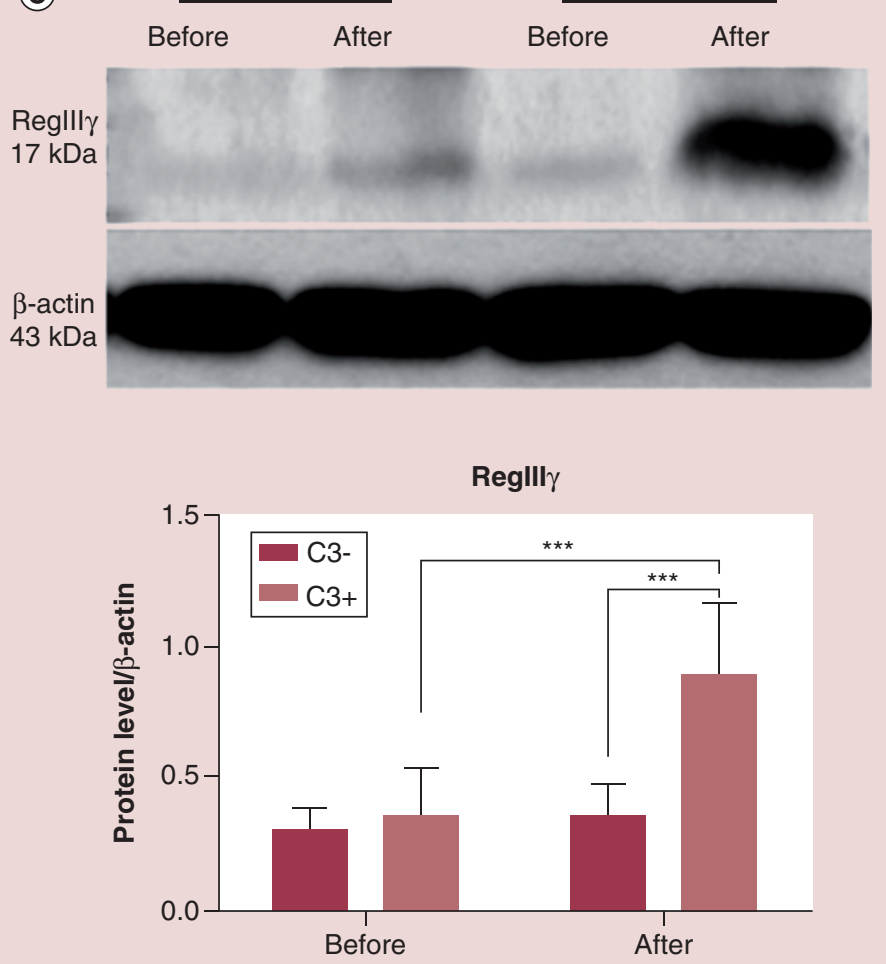

Figure 7. The intracellular activation of $\mathrm{C} 3$ in the isolated Paneth cells after $48 \mathrm{~h}$ of in vitro culturing. Separated PCs were harvested from the bottom crypts of ileum in Sham-operated mice, followed by exogenous C3 stimulation or not. (A) The deposition of C3 related proteins in isolated PCs was compared between the C3- group and the C3+ group via the Western blot method. ${ }^{* *} \mathrm{p}<0.01,{ }^{* * *} \mathrm{p}$ $<0.001$ versus C3- group. (B) The production of PAP III (labeled with anti-PAP-III, green) in PCs after $48 \mathrm{~h}$ of culturing via the immunofluorescence method. The yellow arrows indicate nuclei of PCs (stained with DAPI, blue). Scale bars, $10 \mu \mathrm{m}$. (C) The production of RegIII- $\gamma$ in PCs before and after the culture with or without C3 via the Western blot method. The amount of Reglll- $\gamma$ protein was compared with histograms. ${ }^{* *} p<0.001$ versus C3- group. Representative histograms of $n=5$ independent experiments. DAPI: 4'6-diamidino-2-phenylindole; PC: Paneth cell.

or absorption of exogenous human C3. Either murine antibody or detection of C3 mRNA expression should be applied to determine whether PCs are being stimulated to produce C3 intracellularly. To our knowledge, purified mouse C3 reagent is hard to produce in large volume, and the usage of alternative human C3 is successfully reported in earlier studies [40,41]. Our works suggest that intracellular C3 may play valuable roles in regulation of PCs during AGI. However, there is another concern that a possible impact of C3 immunotherapy is to help control of abdominal infection, rather than stimulation of PCs. Indeed, our previous study has observed a reduction in severity of regional bacterial burden due to exogenous C3 treatment [8]. It is well recognized that inflammation and infection are linked to wound healing processes in most tissues. Moreover, recent studies have confirmed that both C3 activation and PCs played vital functions in promoting wound healing far beyond immunity [27,32,42-44]. Along with a direct connection between $\mathrm{C} 3$ and PCs in vitro experiments, our works show that C3 immunotherapy would play valuable roles in mediating injury healing beyond infection control during AGI.

\section{Conclusion}

Massive C3 deposition and activation in intestinal epithelial cells are responsible for AGI. Exogenous C3 treatment in early stage of abdominal infection could inhibit such deposition in intestinal epithelium possibly by enhancing the function and proliferation of PCs during an acute injury. The intracellular activation of C3 detected in vitro PCs might be responsible for their proliferation and subsequent injury-healing promotion, which should be further validated in vivo during the course of AGI. 


\section{Summary points}

- This research aimed to explore the role of complement C3 activation inside Paneth cells (PCs) in promoting the repair of gut epithelia during an acute gastrointestinal injury (AGI).

- All mice suffered from abdominal sepsis to induce extensive intestinal injury and received various C3 interventions in early stage of acute injury.

- AGI resulted in a high mortality, while C3 therapy significantly attenuated epithelial damages and improved survival. Moreover, exogenous C3 treatment enhanced functions of PCs in vitro through intracellular activation during an early stage of injury.

- C3 immunotherapy might play a valuable role in gut epithelial repair during AGI by regulating C3 intracellular activation in PCs.

Meeting presentation

RCSEd/CSHK Conjoint Scientific Congress 2018, 15-16 September 2018. College's 104th Clinical Congress, ACS, Boston, Massachusetts, 21-25 October 2018.

\section{Acknowledgments}

We thank Shenyan Wu and American Journal Expert ${ }^{\complement}$ for language polishing works of this manuscript.

\section{Author contributions}

Y Yuan made substantial contributions to the conception or design of the work. J Zhang, J Ye, Y Ren and J Zuo carried out the experiments. Y Yuan and M Tan analyzed the data for the work. J Zhang, J Ye, Y Ren and Y Yuan drafted the manuscript. Y He and Y Yuan revised paper critically for important intellectual content. Y Yuan did final approval of the version to be published. J Zhang and $Y$ Yuan were agreement to be accountable for all aspects of the work in ensuring that questions related to the accuracy or integrity of any part of the work are appropriately investigated and resolved.

Financial \& competing interest disclosure

This work was supported by grants from National Natural Science Foundation of China (number 81401307) and Medical Science and Technology Research Fund Project in Guangdong province (A2015112). The authors have no other relevant affiliations or financial involvement with any organization or entity with a financial interest in or financial conflict with the subject matter or materials discussed in the manuscript apart from those disclosed.

No writing assistance was utilized in the production of this manuscript.

Ethical conduct

The authors have obtained appropriate institutional review board approval or have followed the principles outlined in the Declaration of Helsinki for all human or animal experimental investigations. All the experiments were approved by the administration of the Committee of Experimental Animals in the First Affiliated Hospital of Sun Yat-sen University, PR China. All animal experiments were carried out in accordance with international standards on animal welfare and the China Animal Welfare Legislation. Efforts were made to minimize the number of animals used and their suffering.

\section{Open access}

This work is licensed under the Attribution-NonCommercial-NoDerivatives 4.0 Unported License. To view a copy of this license, visit http://creativecommons.org/licenses/by-nc-nd/4.0/

\section{References}

1. Blikslager AT, Moeser AJ, Gookin JL, Jones SL, Odle J. Restoration of barrier function in injured intestinal mucosa. Physiol. Rev. 87(2), 545-564 (2007).

2. Yuan Y, Ren J, Wu X, Cao S, Li J. Exogenous C3 postpones complement exhaustion and confers organ protection in murine sepsis. J. Surg. Res. 168(1), e87-e94 (2011).

3. Satyam A, Kannan L, Matsumoto $\mathrm{N}$ et al. Intracellular activation of complement 3 is responsible for intestinal tissue damage during mesenteric ischemia. J. Immunol. 198(2), 788-797 (2017).

4. Tendler DA. Acute intestinal ischemia and infarction. Semin. Gastrointest. Dis. 14(2), 66-76 (2003).

5. Ricklin D, Lambris JD. Complement in immune and inflammatory disorders: pathophysiological mechanisms. J. Immunol. 190(8), 3831-3838 (2013). 
6. Daha MR, Van Kooten C, Roos A. Compliments from complement: a fourth pathway of complement activation? Nephrol. Dial. Transplant 21(12), 3374-3376 (2006).

7. Leung VW, Yun S, Botto M et al. Decay-accelerating factor suppresses complement $\mathrm{c} 3$ activation and retards atherosclerosis in low-density lipoprotein receptor deficient mice. Am. J. Pathol. 175(4), 1757 -1767 ( 2009).

8. Yuan Y, Ren J, Gu G, Cao S, Li J. The effect of human complement C3 protein applied at different times in treatment of polymicrobial sepsis. Inflamm. Res. 61(6), 581-589 (2012).

9. Clevers H. The Paneth cell, caloric restriction, and intestinal integrity. N. Engl. J. Med. 367(16), 1560-1561 (2012).

10. Ayabe T, Satchell DP, Wilson CL, Parks WC, Selsted ME, Ouellette AJ. Secretion of microbicidal alpha-defensins by intestinal Paneth cells in response to bacteria. Nat. Immunol. 1(2), 113-118 (2000).

11. Vaishnava S, Behrendt CL, Ismail AS, Eckmann L, Hooper LV. Paneth cells directly sense gut commensals and maintain homeostasis at the intestinal host-microbial interface. Proc. Natl Acad. Sci. USA 105(52), 20858-20863 (2008).

12. Clevers HC, Bevins CL. Paneth cells: maestros of the small intestinal crypts. Annu. Rev. Physiol. 75, 289-311 (2013).

13. Kopf M, Abel B, Gallimore A, Carroll M, Bachmann MF. Complement component C3 promotes T-cell priming and lung migration to control acute influenza virus infection. Nat. Med. 8(4), 373-378 (2002).

14. Liszewski MK, Kolev M, Le Friec G et al. Intracellular complement activation sustains $\mathrm{T}$ cell homeostasis and mediates effector differentiation. Immunity 39(6), 1143-1157 (2013).

15. Minton K. Innate immunity: the inside story on complement activation. Nat. Rev. Immunol. (14(2), 61 (2013).

16. Zhang $\mathrm{H}$, Cui Z, Luo $\mathrm{G}$ et al. Ghrelin attenuates intestinal ischemia/reperfusion injury in mice by activating the mTOR signaling pathway. Int. J. Mol. Med. 32(4), 851-859 (2013).

17. Collard CD, Montalto MC, Reenstra WR, Buras JA, Stahl GL. Endothelial oxidative stress activates the lectin complement pathway: role of cytokeratin 1. Am. J. Pathol. 159(3), 1045-1054 (2001).

18. Yilmaz OH, Katajisto P, Lamming DW et al. mTORC1 in the Paneth cell niche couples intestinal stem-cell function to calorie intake. Nature 486(7404), 490-495 (2012).

19. Hodin CM, Lenaerts K, Grootjans J et al. Starvation compromises Paneth cells. Am. J. Pathol. 179(6), 2885-2893 (2011).

20. Al Alam D, Danopoulos S, Schall K et al. Fibroblast growth factor 10 alters the balance between goblet and Paneth cells in the adult mouse small intestine. Am. J. Physiol. Gastrointest. Liver Physiol. 308(8), G678-G690 (2015).

21. Rocha JD, Schlossmacher MG, Philpott DJ. LRRK2 and Nod2 promote lysozyme sorting in Paneth cells. Nat. Immunol. 16(9), 898-900 (2015).

22. Chairatana P, Nolan EM. Defensins, lectins, mucins, and secretory immunoglobulin A: microbe-binding biomolecules that contribute to mucosal immunity in the human gut. Crit. Rev. Biochem. Mol. Biol. 52(1), 45-56 (2017).

23. Nieto N, Torres MI, Rios A, Gil A. Dietary polyunsaturated fatty acids improve histological and biochemical alterations in rats with experimental ulcerative colitis. J. Nutr. 132(1), 11-19 (2002).

24. Ehrnthaller C, Ignatius A, Gebhard F, Huber-Lang M. New insights of an old defense system: structure, function, and clinical relevance of the complement system. Mol. Med. 17(3-4), 317-329 (2011).

25. Wagner E, Frank MM. Therapeutic potential of complement modulation. Nat. Rev. Drug. Discov. 9(1), 43-56 (2010).

26. Liszewski MK, Elvington M, Kulkarni HS, Atkinson JP. Complement's hidden arsenal: new insights and novel functions inside the cell. Mol. Immunol. 84, 2-9 (2017).

27. Zhang C, Wang C, Li Y et al. Complement C3a signaling facilitates skeletal muscle regeneration by regulating monocyte function and trafficking. Nat. Commun. 8(1), 2078 (2017).

28. Reichhardt MP, Meri S. Intracellular complement activation-An alarm raising mechanism? Semin. Immunol. doi:10.1016/j.smim.2018.03.003 (2018) (Epub ahead of print).

29. Kulkarni HS, Frakouh C, Byers DE, Brody SL, Gelman AE, Atkinson JP. Intracellular C3 protects human airway epithelial cells from oxidant-stress induced apoptosis: implications for lung allograft injury. J. Heart Lung Transplant. 37(4), S215-S215 (2018).

30. Sato T, Van Es JH, Snippert HJ et al. Paneth cells constitute the niche for Lgr5 stem cells in intestinal crypts. Nature 469(7330), 415-418 (2011).

31. Schmitt M, Schewe M, Sacchetti A et al. Paneth cells respond to inflammation and contribute to tissue regeneration by acquiring stem-like features through SCF/c-Kit signaling. Cell Rep. 24(9), 2312e2317-2328e2317 (2018).

32. Schewe M, Fodde R. Multitasking Paneth cells in the intestinal stem cell niche. In: Intestinal Stem Cell Niche. Bonnet D (Ed.). Elsevier Amsterdam, Netherlands, 41-75 (2018).

33. Sunderhauf A, Skibbe K, Preisker S et al. Regulation of epithelial cell expressed C3 in the intestine - relevance for the pathophysiology of inflammatory bowel disease? Mol. Immunol. 90, 227-238 (2017).

34. Gonzalez LM, Moeser AJ, Blikslager AT. Animal models of ischemia-reperfusion-induced intestinal injury: progress and promise for translational research. Am. J. Physiol. Gastrointest. Liver Physiol. 308(2), G63-G75 (2015). 
35. Ioannou A, Dalle Lucca J, Tsokos GC. Immunopathogenesis of ischemia/reperfusion-associated tissue damage. Clin. Immunol. 141(1), 3-14 (2011).

36. Fleming SD, Mastellos D, Karpel-Massler G, Shea-Donohue T, Lambris JD, Tsokos GC. C5a causes limited, polymorphonuclear cell-independent, mesenteric ischemia/reperfusion-induced injury. Clin. Immunol. 108(3), 263-273 (2003).

37. Fleming SD, Shea-Donohue T, Guthridge JM et al. Mice deficient in complement receptors 1 and 2 lack a tissue injury-inducing subset of the natural antibody repertoire. J. Immunol. 169(4), 2126-2133 (2002).

38. Burger E, Araujo A, Lopez-Yglesias A et al. Loss of Paneth cell autophagy causes acute susceptibility to toxoplasma gondii-mediated inflammation. Cell Host Microbe 23(2), 177e174-190e174 (2018).

39. Riba A, Olier M, Lacroix-Lamande $S$ et al. Paneth cell defects induce microbiota dysbiosis in mice and promote visceral hypersensitivity. Gastroenterology 153(6), 1594e1592-1606e1592 (2017).

40. Prodeus AP, Zhou X, Maurer M, Galli SJ, Carroll MC. Impaired mast cell-dependent natural immunity in complement C3-deficient mice. Nature 390(6656), 172-175 (1997).

41. Fischer WH, Hugli TE. Regulation of B cell functions by C3a and C3a(desArg): suppression of TNF-alpha, IL-6, and the polyclonal immune response. J. Immunol. 159(9), 4279-4286 (1997).

42. Korkmaz HI, Krijnen PaJ, Ulrich MMW, De Jong E, Van Zuijlen PPM, Niessen HWM. The role of complement in the acute phase response after burns. Burns 43(7), 1390-1399 (2017).

43. Hajishengallis G, Reis ES, Mastellos DC, Ricklin D, Lambris JD. Novel mechanisms and functions of complement. Nat. Immunol. 18(12), 1288-1298 (2017).

44. Andersson-Rolf A, Zilbauer M, Koo BK, Clevers H. Stem cells in repair of gastrointestinal epithelia. Physiology (Bethesda) 32(4), 278-289 (2017). 